\title{
Oxygen isotopic ratios in galactic clouds along the line of sight towards Sagittarius B2^
}

\author{
E. T. Polehampton ${ }^{1}$, J.-P. Baluteau ${ }^{2}$, and B. M. Swinyard ${ }^{3}$ \\ 1 Max-Planck-Institut für Radioastronomie, Auf dem Hügel 69, 53121 Bonn, Germany \\ e-mail: epoleham@mpifr-bonn.mpg.de \\ 2 Laboratoire d'Astrophysique de Marseille, CNRS \& Université de Provence, BP 8, 13376 Marseille Cedex 12, France \\ e-mail: Jean-Paul.Baluteau@oamp.fr \\ 3 Rutherford Appleton Laboratory, Chilton, Didcot, Oxfordshire, OX11 0QX, UK \\ e-mail: B.M. Swinyard@rl.ac.uk
}

Received 21 January 2005 / Accepted 31 March 2005

\begin{abstract}
As an independent check on previous measurements of the isotopic abundance of oxygen through the Galaxy, we present a detailed analysis of the ground state rotational lines of ${ }^{16} \mathrm{OH}$ and ${ }^{18} \mathrm{OH}$ in absorption towards the giant molecular cloud complex, Sagittarius B2. We have modelled the line shapes to separate the contribution of several galactic clouds along the line of sight and calculate ${ }^{16} \mathrm{OH} /{ }^{18} \mathrm{OH}$ ratios for each of these features. The best fitting values are in the range 320-540, consistent with the previous measurements in the Galactic Disk but slightly higher than the standard ratio in the Galactic Centre. They do not show clear evidence for a gradient in the isotopic ratio with galactocentric distance. The individual ${ }^{16} \mathrm{OH}$ column densities relative to water give ratios of $\left[\mathrm{H}_{2} \mathrm{O} / \mathrm{OH}\right]=0.6-1.2$, similar in magnitude to galactic clouds in the sight lines towards W51 and W49. A comparison with $\mathrm{CH}$ indicates $[\mathrm{OH} / \mathrm{CH}]$ ratios higher than has been previously observed in diffuse clouds. We estimate $\mathrm{OH}$ abundances of $10^{-7}-10^{-6}$ in the line of sight features.
\end{abstract}

Key words. infrared: ISM - ISM: molecules - Galaxy: abundances - ISM: individual objects: Sagittarius B2

\section{Introduction}

The $\mathrm{OH}$ radical is one of the key oxygen bearing species in the interstellar medium (ISM). Not only is it a good diagnostic of physical and chemical conditions (e.g. Goicoechea et al. 2005), but in addition, it provides a very good way to investigate the relative abundance of oxygen isotopes via its isotopologues ${ }^{16} \mathrm{OH},{ }^{18} \mathrm{OH}$ and ${ }^{17} \mathrm{OH}$. Chemical fractionation reactions that might distort the oxygen isotopic ratios in molecular species are not thought to be important (Langer et al. 1984) and this means that observations of $\mathrm{OH}$ can be used directly to determine the ratios ${ }^{16} \mathrm{O} /{ }^{18} \mathrm{O}$ and ${ }^{18} \mathrm{O} /{ }^{17} \mathrm{O}$.

These values are important as they are set by stellar processing and outflow mechanisms and so constrain models of galactic chemical evolution (e.g. Prantzos et al. 1996). ${ }^{16} \mathrm{O}$ is a primary product of stellar nucleosynthesis, produced directly from the primordial elements $\mathrm{H}$ and $\mathrm{He}$ (see Wilson \& Matteucci 1992). Both ${ }^{17} \mathrm{O}$ and ${ }^{18} \mathrm{O}$ are secondary products which require heavier elements from previous nuclear burning for their production. Chemical evolution models (e.g. Prantzos et al. 1996) show that the primary/secondary ratios, ${ }^{16} \mathrm{O} /{ }^{17} \mathrm{O}$ and ${ }^{16} \mathrm{O} /{ }^{18} \mathrm{O}$, should fall with decreasing galactocentric

^ Based on observations with ISO, an ESA project with instruments funded by ESA Member States (especially the PI countries: France, Germany, The Netherlands and the UK) with the participation of ISAS and NASA. distance due to the increased processing rate towards the Galactic Centre. These ratios should also fall with time due to the build up of the secondary elements in the ISM.

Previous measurements of ${ }^{16} \mathrm{O} /{ }^{18} \mathrm{O}$ in the ISM have mainly been made via the radio lines of $\mathrm{H}_{2} \mathrm{CO}$ and $\mathrm{CO}$ towards molecular clouds (see Wilson \& Rood 1994). However, these lines are generally optically thick in the most abundant isotopologues and so double ratios such as $\mathrm{H}_{2}^{13} \mathrm{C}^{16} \mathrm{O} / \mathrm{H}_{2}^{12} \mathrm{C}^{18} \mathrm{O}$ are used. This relies on accurate knowledge of ${ }^{12} \mathrm{C} /{ }^{13} \mathrm{C}$ which is subject to chemical fractionation in molecular species (e.g. Langer et al. 1984).

A very good way to get around these difficulties is to use the $\mathrm{OH}$ molecule - several measurements using its $18 \mathrm{~cm}$ $\Lambda$-doubling transitions have been made towards the Galactic Centre (Whiteoak \& Gardner 1981; Williams \& Gardner 1981), although in some circumstances these can be complicated by excitation effects within the hyperfine levels (Bujarrabal et al. 1983). This problem can be avoided by using the farinfrared (FIR) rotational lines which should not be affected by hyperfine excitation anomalies. These provide an excellent way to independently check the ratios determined at radio wavelengths.

Rotational transitions of $\mathrm{OH}$ have previously been studied towards the Orion Kleinmann-Low nebula using the Kuiper Airborne Observatory (Melnick et al. 1990, and references 
therein) where both ${ }^{16} \mathrm{OH}$ and ${ }^{18} \mathrm{OH}$ were used to constrain the physical conditions of the source. More recently, Goicoechea \& Cernicharo (2002) have used the Infrared Space Observatory (ISO) satellite to observe $\mathrm{OH}$ in the envelope of the giant molecular cloud complex Sagittarius B2 (Sgr B2). The lowest energy transitions show absorption due to both ${ }^{16} \mathrm{OH}$ and ${ }^{18} \mathrm{OH}$ in Sgr B2 itself and in foreground features intersecting the line of sight. They indicate that the isotopic ratios are broadly similar to the previous radio results.

In this paper we conduct a more detailed investigation into ISO observations towards Sgr B2, with the aim of separating the relative abundances of ${ }^{16} \mathrm{OH}$ and ${ }^{18} \mathrm{OH}$ in individual absorption components in the line of sight. A similar comparison of ${ }^{18} \mathrm{OH}$ with ${ }^{17} \mathrm{OH}$ has already been presented by Polehampton et al. (2003). We have used data from a wide spectral survey carried out with the ISO Long Wavelength Spectrometer (LWS; Clegg et al. 1996) Fabry-Pérot mode. A combination of prime and non-prime data from the survey allowed us to increase the signal-to-noise ratio over the standard data and derive an accurate and consistent calibration for two ground state transitions of ${ }^{16} \mathrm{OH}$ and one transition of ${ }^{18} \mathrm{OH}$.

After presenting the observations and results, we describe a model of the line shape to separate the line of sight absorption into 10 velocity components (Sect. 4). In Sect. 5 we assign the ${ }^{16} \mathrm{OH} /{ }^{18} \mathrm{OH}$ ratio for each component to a galactocentric distance and compare with previous determinations of the isotopic abundances. We discuss the final ${ }^{16} \mathrm{OH}$ column densities in relation to other related species in Sect. 6.

\section{Observations and data reduction}

Sgr B2 was observed as part of a wide spectral survey using the ISO LWS Fabry-Pérot (FP) mode L03. Unbiased coverage of the whole LWS spectral range $(47-196 \mu \mathrm{m})$ was carried out using 36 separate observations with a spectral resolution of $30-40 \mathrm{~km} \mathrm{~s}^{-1}$. No other object outside of the Solar System was observed over the complete LWS spectral range in this way. Results from this survey have been presented by Ceccarelli et al. (2002), Polehampton et al. (2002a), Vastel et al. (2002), Polehampton et al. (2003), Polehampton et al. (2005). Sgr B2 was also extensively observed by the LWS FP in narrow wavelength scans using the L04 mode (see Goicoechea et al. 2004). We have used several of these L04 observations to improve the signal-to-noise ratio in the L03 data for ${ }^{18} \mathrm{OH}$. The ISO TDT numbers for all the observations used in this paper are detailed in Table A.1.

The LWS beam had an effective diameter of approximately 80" (Gry et al. 2003) and L03 observations were pointed at coordinates $\alpha=17^{\mathrm{h}} 47^{\mathrm{m}} 21.75^{\mathrm{s}}, \delta=-28^{\circ} 23^{\prime} 14.1^{\prime \prime}$ (J2000). This gave the beam centre an offset of 21.5" from the main FIR peak at Sgr B2 (M) - this pointing was used to exclude the source Sgr B2 $(\mathrm{N})$ from the beam. The additional L04 observations were pointed directly towards the nominal position of Sgr B2 (M), but careful comparison showed no significant difference to the L03 observations. Each observation had a spectral sampling interval of $1 / 4$ resolution element with each point repeated 3-4 times in L03 mode and 11-15 times in L04 mode.

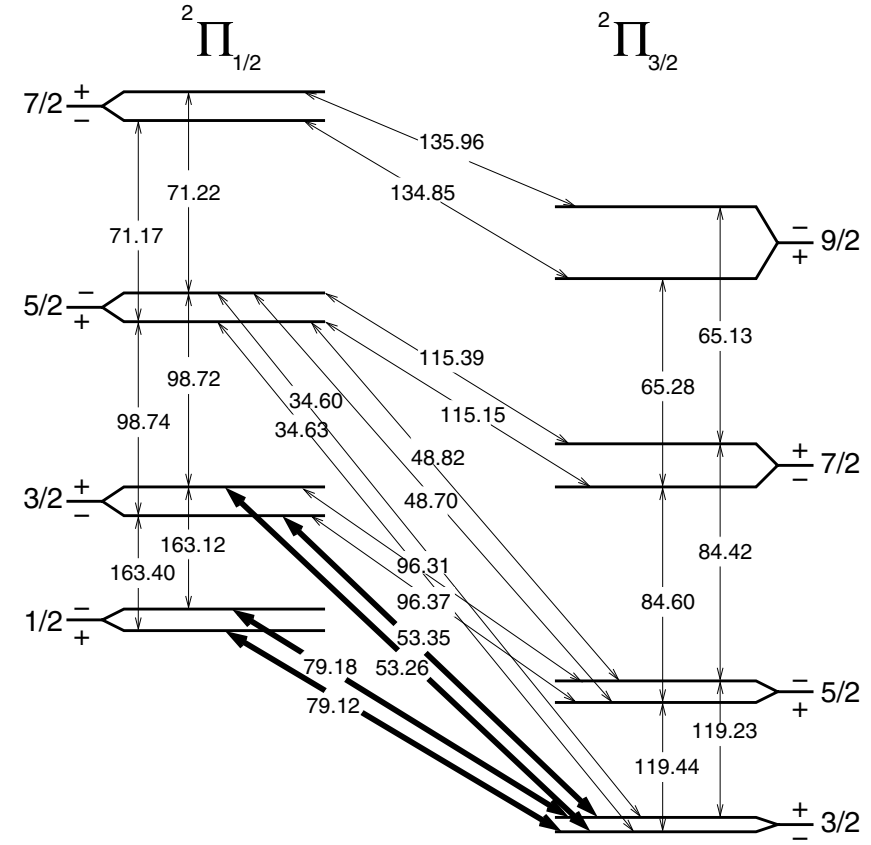

Fig. 1. Low lying rotational energy levels in the electronic and vibrational ground state of the ${ }^{16} \mathrm{OH}$ molecule, adapted from Brown et al. (1982). The $\Lambda$-doubling of each level is shown but the splitting has been exaggerated for clarity. The relative spacing of the levels in energy is only approximate. The $J$ values for each level are shown and the wavelength of each transition is given in $\mu \mathrm{m}$. The transitions used in the analysis presented here are shown as thick arrows.

In addition to the primary spectral survey data, each line was generally included in at least one other L03 observation. This occurred because the LWS used 10 detectors, which always recorded data in their own spectral ranges (the prime observations consist of data from a single detector for which instrument settings were optimised). We have included these "non-prime" data to further improve the signal-to-noise ratio.

Each observation was carefully reduced using the LWS offline pipeline (OLP) version 8 (for FP data the difference between OLP version 8 and the latest version 10 is not significant). Further processing was then carried out interactively using routines that appeared in the LWS Interactive Analysis package version 10 (LIA10: Lim et al. 2002) and the ISO Spectral Analysis Package (ISAP: Sturm et al. 1998). The method included determination of accurate dark currents (including stray light), interactive division of each mini-scan by the LWS grating response profiles and careful scan by scan removal of glitches (see Polehampton et al. 2002a, 2003).

Before co-adding the data for each line, the wavelength scale of each observation was corrected to the local standard of rest (LSR). Non-prime observations were carefully checked against the equivalent prime measurement to align the line centres. This is particularly important for non-prime data measured with the LWS long wavelength FP (FPL) below $70 \mu \mathrm{m}$ (outside its nominal operating range) because the standard FPL wavelength calibration did not include short wavelength data (Gry et al. 2003). For the ${ }^{16} \mathrm{OH} J=3 / 2-3 / 2$ cross ladder transition at $53 \mu \mathrm{m}$ (Fig. 1 shows the energy levels for $\mathrm{OH}$ ), we have used only non-prime FPL data. This is because it has a significantly 


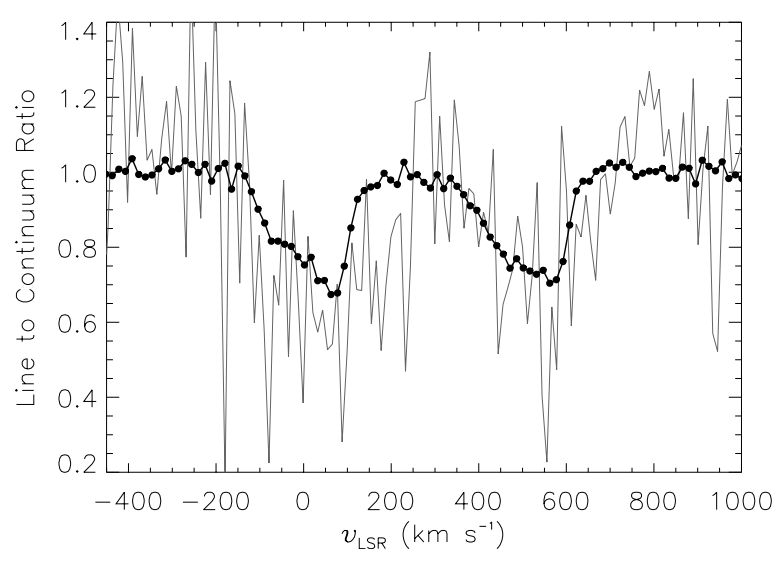

Fig. 2. The ${ }^{16} \mathrm{OH}^{2} \Pi_{1 / 2}{ }^{2} \Pi_{3 / 2} J=3 / 2-3 / 2$ transition at $53.26 \mu \mathrm{m}$ from non-prime data (black) and prime data (grey). Both datasets are binned at $1 / 4$ of their respective resolution elements (non-prime: $61 \mathrm{~km} \mathrm{~s}^{-1}$ and prime: $45 \mathrm{~km} \mathrm{~s}^{-1}$ ). The gain in signal-to-noise in the non-prime data is a factor of $\sim 9$.

higher signal-to-noise ratio than the prime data (due to the higher transmission of FPL compared to the short wavelength FP, FPS; Polehampton et al. 2002b). The absolute wavelength alignment was difficult to determine purely by comparison with the noisy prime data and so a velocity shift was allowed as a free parameter in the modelling (see Sect. 4.1). Figure 2 shows the resulting shift cross-checked with the prime data. The gain in signal-to-noise achieved by using FPL rather than FPS is approximately a factor of 9 . The only cost in using these data is a reduction in spectral resolution from $45 \mathrm{~km} \mathrm{~s}^{-1}$ (for FPS) to $61 \mathrm{~km} \mathrm{~s}^{-1}$ (for FPL).

After co-addition, the continuum around each line was fitted with a 3rd order polynomial baseline which was then divided into the data to obtain the relative depth of the lines below the continuum. This effectively bypassed the large systematic uncertainties in the multiplicative calibration factors needed to obtain the absolute flux scale (see Swinyard et al. 1998). The remaining errors are due to detector noise, uncertainty in the dark current and the polynomial fit.

At the resolution of the LWS, two components are visible for each $\mathrm{OH}$ transition due to the $\Lambda$-doublet type splitting of each rotational level. However, further hyperfine splitting is not resolved. The two $\Lambda$-doublet components showed good agreement in the data, and so where they were well separated (the $J=3 / 2-3 / 2$ cross ladder transition at $53 \mu \mathrm{m}$ for ${ }^{16} \mathrm{OH}$ and the $J=5 / 2-3 / 2$ transition at $120 \mu \mathrm{m}$ for ${ }^{18} \mathrm{OH}$ ), they were co-added to further increase the signal-to-noise ratio.

\section{Results}

Figure 1 shows the low-lying rotational transitions of ${ }^{16} \mathrm{OH}$, most of which are included in the spectral survey range. The strongest lines observed in the survey were due to the ${ }^{2} \Pi_{3 / 2} J=$ $5 / 2-3 / 2$ transition from the ground state at $119 \mu \mathrm{m}$. These lines show almost complete absorption of the FIR continuum in the range -150 to $+100 \mathrm{~km} \mathrm{~s}^{-1}$ (see Goicoechea \& Cernicharo 2002). Due to the large depth of the lines, the shape was strongly affected by the transient response of the LWS detectors. These detector memory effects (see Lloyd 2002) meant that successive repeated scans underestimated the depth and the continuum level following each line. Therefore, the lines were not used in the analysis presented here. There are two remaining transitions from the ground rotational state (see Fig. 1) that occur between the ${ }^{2} \Pi_{3 / 2}$ and ${ }^{2} \Pi_{1 / 2}$ ladders: $J=1 / 2-3 / 2$ at $79 \mu \mathrm{m}$ and $J=3 / 2-3 / 2$ at $53 \mu \mathrm{m}$. These lines are also broad but with much lower optical depth. They are shown in Fig. 3. The broad absorption is due to features between the Sun and Galactic Centre associated with galactic spiral arms that cross the line of sight (e.g. Greaves \& Williams 1994).

Several ${ }^{16} \mathrm{OH}$ transitions between higher energy rotational levels are observed in the survey at the velocity of Sgr B2 itself but do not occur in the line of sight clouds. These lines originate in the envelope of Sgr B2 and have been modelled by Goicoechea \& Cernicharo (2002). They show that the excited $\mathrm{OH}$ originates in clumpy photodissociation regions (PDRs) on the edge of the Sgr B2 complex at temperatures of 40-600 K. In this paper, we concentrate on the broad absorption observed in the ground state lines at 79 and $53 \mu \mathrm{m}$.

Due to the lower abundance of ${ }^{18} \mathrm{OH}$, the fundamental ground state ${ }^{2} \Pi_{3 / 2} J=5 / 2-3 / 2$ transition is much weaker and the cross-ladder and higher energy lines are not detected. Figure 4 shows the $J=5 / 2-3 / 2$ line after co-adding the two resolved $\Lambda$-doublet components. These observations were previously presented by Polehampton et al. (2003). Here we have used additional data from the L04 mode, which showed good agreement with the L03 data.

\section{Modelling of $\mathrm{OH}$ in the line of sight}

\subsection{High resolution model}

At the spectral resolution of the LWS, the line of sight components are blended together into a single broad absorption. We have modelled the line shape using higher spectral resolution measurements to fix the velocities and widths of each component. Comparison of high resolution spectra tracing molecular and atomic species in these clouds show very similar line widths and velocities (CO; e.g. Vastel et al. (2002), $\mathrm{H}_{2} \mathrm{CO}$; e.g. Mehringer et al. (1995), HI; e.g. Garwood \& Dickey (1989)). These tracers also show a very similar velocity structure to observations of the $\Lambda$-doubling lines of $\mathrm{OH}$ at cm wavelengths (McGee et al. 1970; Whiteoak \& Gardner 1975, 1981; Williams \& Gardner 1981; Bujarrabal et al. 1983).

We have assumed that the $\mathrm{H}$ I measurements are a representative tracer and based our model on the published parameters from Garwood \& Dickey (1989) (after correcting an error in their Table 2: $11 \mathrm{~km} \mathrm{~s}^{-1}$ should read $1.1 \mathrm{~km} \mathrm{~s}^{-1}$ ). This method has already been used to successfully fit the absorption of $\mathrm{CH}$ and $\mathrm{CH}_{2}$ (Polehampton et al. 2005) and is similar to the method used to fit O I and C II lines by Vastel et al. (2002).

The HI observations were made using the VLA pointed close to Sgr B2 (M). In our model, the H I data were used to fix the velocity and line width of 10 line of sight features with each component assumed to have a Gaussian line shape. The optical depth at each velocity, $\tau$, was then adjusted as a free parameter and the line-to-continuum ratio calculated from,

$I=I_{\mathrm{c}} \exp (-\tau)$ 

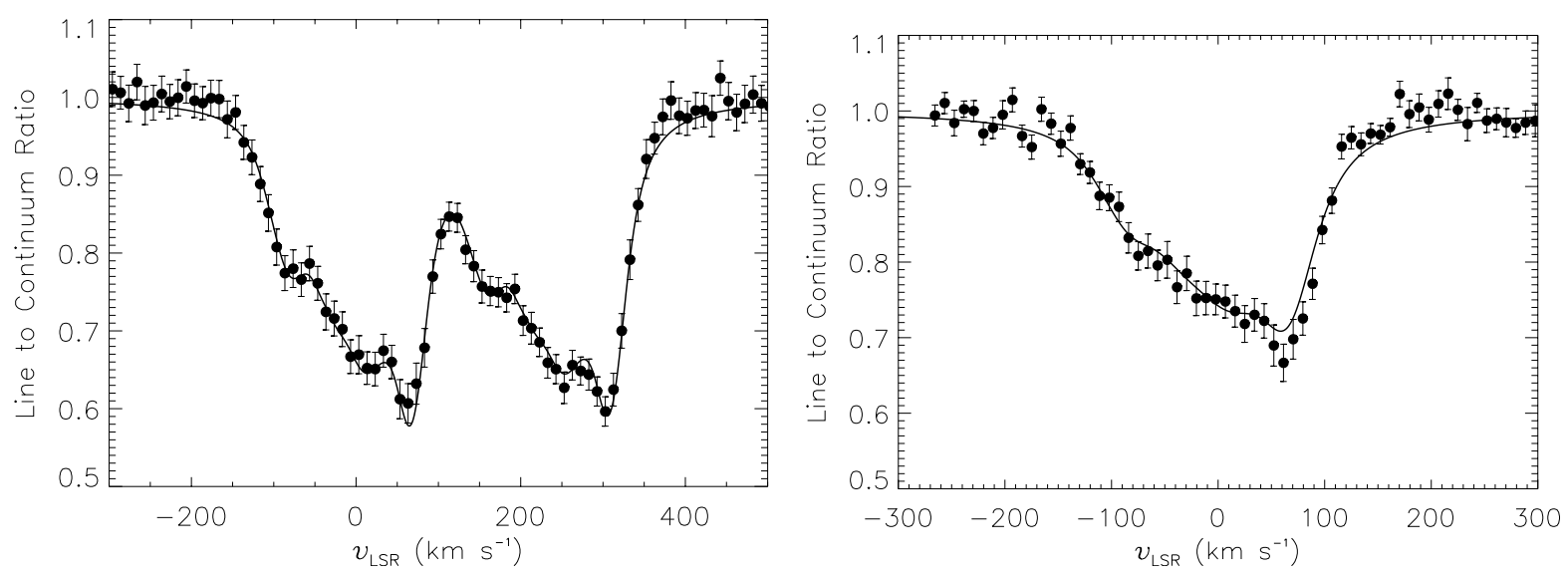

Fig. 3. Data for the ${ }^{16} \mathrm{OH}$ cross ladder $\left({ }^{2} \Pi_{1 / 2}{ }^{2} \Pi_{3 / 2}\right)$ transitions, $J=1 / 2-3 / 2$ at $79 \mu \mathrm{m}$ (left; binned at $1 / 4$ resolution element) and $J=3 / 2-3 / 2$ at $53 \mu \mathrm{m}$ ( right; binned at $\sim 1 / 6$ resolution element), plotted as line to continuum ratio. The errors shown represent the combined statistical and systematic uncertainty in each bin. The two $\Lambda$-doublet components have been averaged for the $53 \mu \mathrm{m}$ line. The best fitting model accounting for the absorbing clouds in the line of sight is shown as the solid line (see text).

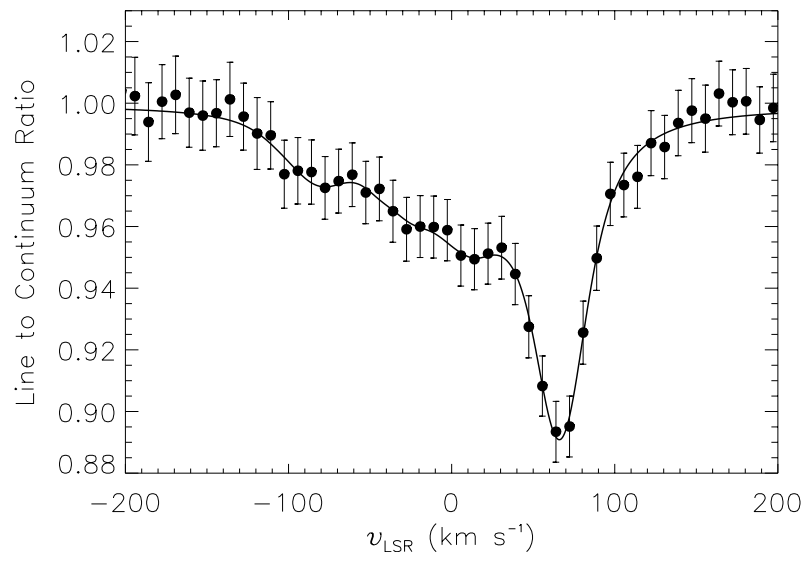

Fig. 4. Co-added data from the two $\Lambda$-doublet components of the ${ }^{18} \mathrm{OH}$ ${ }^{2} \Pi_{3 / 2} J=5 / 2-3 / 2$ transition. The data were binned at $1 / 4$ resolution element and the errors represent the combined statistical and systematic uncertainty in each bin. The best fitting model accounting for absorbing clouds in the line of sight is shown (see text).

where $I_{\mathrm{c}}$ is the intensity of the continuum. The resulting spectrum was then convolved to the resolution of the LWS. In order to put the best constraint on the line shape, the two ${ }^{16} \mathrm{OH}$ lines were fitted simultaneously with the ${ }^{18} \mathrm{OH}$ line. Optical depths were varied in the model for the $79 \mu \mathrm{m}$ line and then used to calculate the shape of $53 \mu \mathrm{m}$ line assuming that both lines trace the same column density. This must be true as they originate in the same lower level. Column densities were calculated assuming a Doppler line profile with Maxwellian velocity distribution (e.g. Spitzer 1978),

$N_{j}=\frac{8 \pi \sqrt{\pi}}{2 \sqrt{\ln 2}} 10^{17} \frac{\tau_{0} \Delta v}{A_{i j} \lambda_{i j}^{3} g_{i} / g_{j}}$

where $N_{j}$ is the column density in the lower level in $\mathrm{cm}^{-2}$, $\tau_{0}$ is the optical depth at line centre, $\Delta v$ is the line width in $\mathrm{km} \mathrm{s}^{-1}, A_{i j}$ is the Einstein coefficient for spontaneous emission in $\mathrm{s}^{-1}, \lambda_{i j}$ is the wavelength in $\mu \mathrm{m}$ and $g_{i}$ is the statistical weight of state $i$. The line wavelengths and Einstein coefficients used (averaged over the unresolved hyperfine structure) are shown in Table A.2.

Further free parameters were added to the fit describing the ${ }^{16} \mathrm{OH} /{ }^{18} \mathrm{OH}$ column density ratio, allowing the shape of the $120 \mu \mathrm{m}{ }^{18} \mathrm{OH}$ line to be calculated. Clouds which are thought to reside at similar galactocentric distances were forced to have identical isotope ratios - this affects the features at $-108 /-82 \mathrm{~km} \mathrm{~s}^{-1},-52 /-44 \mathrm{~km} \mathrm{~s}^{-1}$ and $+53 /+67 \mathrm{~km} \mathrm{~s}^{-1}$. In order to account for any drift in the wavelength calibration between the lines, a free velocity shift was allowed for each line, giving a total of 20 free parameters in the fit. The most important factor constraining the velocity shift was the relatively deep and narrow absorption at the velocity of Sgr B2 in the ${ }^{18} \mathrm{OH}$ line. The Numerical Recipes multi-parameter fitting routine, "amoeba" (Press et al. 1992), was used to minimise $\chi^{2}$.

In order to ensure that the algorithm did not converge at a false minimum, the initial conditions were set close to their best values and the routine was restarted at the first convergence point. Solutions with negative absorption were avoided by setting $\chi^{2}$ to be high when the optical depth went below zero.

The best fitting model is shown plotted with the data for each line in Figs. 3 and 4. The final ${ }^{16} \mathrm{OH}$ column densities and ${ }^{16} \mathrm{OH} /{ }^{18} \mathrm{OH}$ ratios are shown in Table 1 . In the line of sight clouds where no absorption is observed from higher energy levels (Goicoechea \& Cernicharo 2002), the ground state population is a good measure of the total $\mathrm{OH}$ column density. The fitted components are all optically thin except at the velocity of Sgr B2 itself, where optical depths of 2.5-3.3 were found for the ${ }^{16} \mathrm{OH}$ lines. However, these are not high enough to break the assumption of purely Doppler line profiles used in the model and calculation of column densities.

The best fitting velocity shift applied to the ${ }^{16} \mathrm{OH} J=$ $1 / 2-3 / 2$ line at $79 \mu \mathrm{m}$ was $8.8 \mathrm{~km} \mathrm{~s}^{-1}$ and for the ${ }^{18} \mathrm{OH} J=5 / 2-3 / 2$ line at $120 \mu \mathrm{m}$ was $-1.9 \mathrm{~km} \mathrm{~s}^{-1}$. These shifts centred the deepest absorption on the H I component at $+67 \mathrm{~km} \mathrm{~s}^{-1}$ and are within the uncertainty in FP wavelength calibration $\left(<11 \mathrm{~km} \mathrm{~s}^{-1}\right.$; Gry et al. 2003). However, for the ${ }^{16} \mathrm{OH} J=3 / 2-3 / 2$ line at $53 \mu \mathrm{m}$ using data taken with nonprime detectors, a larger shift of $28 \mathrm{~km} \mathrm{~s}^{-1}$ was required. This 
Table 1. Column densities and ${ }^{16} \mathrm{OH} /{ }^{18} \mathrm{OH}$ ratios for the best fit carried out to the ${ }^{16} \mathrm{OH}$ and ${ }^{18} \mathrm{OH}$ ground state lines. Columns 1 and 2 show the Gaussian fit parameters from the H I observations of Garwood \& Dickey (1989).

\begin{tabular}{cccc}
\hline \hline $\begin{array}{c}\text { Velocity } \\
\left(\mathrm{km} \mathrm{s}^{-1}\right)\end{array}$ & $\begin{array}{c}F W H M \\
\left(\mathrm{~km} \mathrm{~s}^{-1}\right)\end{array}$ & $\begin{array}{c}N\left({ }^{16} \mathrm{OH}\right) \\
\left(10^{15} \mathrm{~cm}^{-2}\right)\end{array}$ & ${ }^{16} \mathrm{OH} /{ }^{18} \mathrm{OH}$ \\
$-108 \pm 2$ & $7 \pm 4$ & $0.56 \pm 0.39$ & $460_{-50}^{+90}$ \\
$-82 \pm 4$ & $28 \pm 12$ & $9.0 \pm 3.9$ & $460_{-50}^{+90}$ \\
$-44.0 \pm 0.6 /(-52 \pm 6)^{a}$ & $8 \pm 2(17 \pm 5)$ & $3.5 \pm 1.8$ & $450_{-60}^{+100}$ \\
$-24.4 \pm 0.8$ & $14 \pm 2$ & $5.4 \pm 1.8$ & $370_{-50}^{+110}$ \\
$+1.1 \pm 0.4$ & $19 \pm 1$ & $7.6 \pm 3.4$ & $430_{-40}^{+80}$ \\
$+15.7 \pm 0.3$ & $7 \pm 1$ & $3.6 \pm 2.3$ & $360_{-50}^{+80}$ \\
$+31 \pm 4$ & $21 \pm 14$ & $5.4 \pm 3.8$ & $540_{-50}^{+100}$ \\
$+66.7 \pm 0.5 /(+52.8 \pm 0.9)^{b}$ & $16 \pm 1(11 \pm 2)$ & $32_{-4}^{+6}$ & $320_{-30}^{+70}$ \\
\hline Total & \multicolumn{3}{c}{67.0} \\
\hline
\end{tabular}

${ }^{a}$ These two components are too close to separate in our model and the best fit line shape requires only the $-44.0 \mathrm{~km} \mathrm{~s}^{-1}$ component.

${ }^{b}$ The H I data resolve 2 components in Sgr B2. However, our fit requires only one of these (at $66.7 \mathrm{~km} \mathrm{~s}^{-1}$ ) to reproduce the ISO spectrum.

shift is due to the fact that this line was observed with FPL outside of its nominal range (see Sect. 2). In order to determine if this large velocity offset was reasonable, we compared the shifted data to the noisier prime observation (in which the accuracy of the wavelength calibration should be better than $6 \mathrm{~km} \mathrm{~s}^{-1}$; Gry et al. 2003). The two line components show good agreement (see Fig. 2) and the line shift is consistent with that found for other spectral lines observed using FPL below $70 \mu \mathrm{m}$ (Polehampton 2002).

The final fit shows that only 8 of the 10 velocity components are necessary to reproduce the observed line shape. At the velocity associated with Sgr B2, the width of the absorption in the ${ }^{18} \mathrm{OH}$ line is too narrow to allow strong absorption by both the +53 and $+67 \mathrm{~km} \mathrm{~s}^{-1}$ components observed for $\mathrm{H}$ I. This is consistent with the results obtained by fitting the ground state line of $\mathrm{CH}$ (Polehampton et al. 2005). Also, the two components at -52 and $-44 \mathrm{~km} \mathrm{~s}^{-1}$ are too closely spaced to be separated in our fit and significant optical depth was found only at $-44 \mathrm{~km} \mathrm{~s}^{-1}$ (in Table 1, only one value centred at $-44 \mathrm{~km} \mathrm{~s}^{-1}$ is given for the two components).

We have also investigated the effect of further reducing the number of fit components in order to increase confidence in the final column densities and ratios. The minimum number of components that can reasonably be fitted to the spectrum is 4 (e.g. following Neufeld et al. 2000). We re-ran our model using 4 velocity components corresponding to the ranges used by Neufeld et al. and widths estimated from their $\mathrm{H}_{2}^{18} \mathrm{O}$ spectrum observed with the Submillimeter Wave Astronomy Satellite (SWAS). These 4 empirically determined components can broadly reproduce the observed line shapes. The fit gives ${ }^{16} \mathrm{OH}$ column densities and ${ }^{16} \mathrm{OH} /{ }^{18} \mathrm{OH}$ ratios that are within the errors of the results from Table 1 summed in the relevant velocity ranges. The ${ }^{16} \mathrm{OH} /{ }^{18} \mathrm{OH}$ ratios obtained are, 480 (centred at $-80 \mathrm{~km} \mathrm{~s}^{-1}$ ), 470 (centred at $-40 \mathrm{~km} \mathrm{~s}^{-1}$ ), 510 (centred at $+10 \mathrm{~km} \mathrm{~s}^{-1}$ ) and 290 (centred at $+65 \mathrm{~km} \mathrm{~s}^{-1}$ ). However, we have used the high resolution $\mathrm{H}$ I observations as a basis for our model because this can not only fully describe the line shapes but also allows the contribution of clouds at different galactocentric distances along the line of sight to be disentangled.

\subsection{Errors on fitted parameters}

The number of closely spaced velocity components in the fit made the modelling difficult as their separation was less than the resolution of the LWS. This meant that variation in one component could be compensated for by changing another, resulting in a relatively large uncertainty in each fitted optical depth. In addition, the results for each component are likely to be an average over several closely spaced narrow features such as those observed showing CS absorption with velocity widths $\sim 1 \mathrm{~km} \mathrm{~s}^{-1}$ (Greaves \& Williams 1994). However, this would only alter the total column densities if a few of the narrow features had very much higher optical depths than the others, and this does not appear to be the case in the CS data.

In order to estimate the uncertainty on each fitted parameter we performed a Monte-Carlo error analysis. The best fitting model determined by minimising $\chi^{2}$ was used to generate a set of synthetic spectra where each point had a mean value equal to the best fit and standard deviation equal to the original data error. We re-fitted each synthetic spectrum using the original fitting method and analysed the resulting dataset for each parameter. The results show that the errors on neighbouring optical depths are strongly correlated, but that these are not correlated with the uncertainty in ${ }^{16} \mathrm{OH} /{ }^{18} \mathrm{OH}$ ratios (except for the Sgr B2 component). This is due to the fact that the best fit isotopologue ratios depend more on the overall line shape than on the relative optical depth in neighbouring components. The final uncertainty in column density is shown in Table 1 as a combination of $1 \sigma$ errors in H I line width (as quoted by Garwood \& Dickey 1989) and the modelling errors in optical depth. The errors in ${ }^{16} \mathrm{OH} /{ }^{18} \mathrm{OH}$ ratio were determined directly from the Monte-Carlo analysis.

\subsection{Comparison with previous results}

The column density at the velocity of Sgr B2 has previously been calculated by Goicoechea \& Cernicharo (2002) using ISO FP data observed in the L04 mode. They used a radiative transfer model and accounted for the populations in 
higher energy levels up to $420 \mathrm{~K}$ above ground to determine $N\left({ }^{16} \mathrm{OH}\right)=(1.5-2.5) \times 10^{16} \mathrm{~cm}^{-2}$. Lower resolution observations with the LWS grating mode show that $\mathrm{OH}$ is widespread across the whole Sgr B2 region with column densities in the range (2-5) $\times 10^{16} \mathrm{~cm}^{-2}$ (Goicoechea et al. 2004). Our value associated with Sgr B2 from Table 1 gives $N\left({ }^{16} \mathrm{OH}\right)=\left(3.2_{-0.4}^{+0.6}\right) \times$ $10^{16} \mathrm{~cm}^{-2}$ in the ground state level. This is slightly higher than the previous FP result but fits into the general picture reasonably well. We also find a slightly higher value than Goicoechea \& Cernicharo $(2002)$ for $N\left({ }^{18} \mathrm{OH}\right):(9.8 \pm 1.6) \times 10^{13} \mathrm{~cm}^{-2} \mathrm{com}-$ pared to $(6 \pm 2) \times 10^{13} \mathrm{~cm}^{-2}$.

The column density of ${ }^{16} \mathrm{OH}$ has also been measured from observations of its $\Lambda$-doubling lines at $18 \mathrm{~cm}$. Bieging (1976) used the interferometer of the Owens Valley Radio Observatory (OVRO) to calculate an integrated column density over the velocity range $40-89 \mathrm{~km} \mathrm{~s}^{-1}$ equal to $1.2 \times 10^{17} \mathrm{~cm}^{-2}$. This would indicate that the FIR lines underestimate the column density by a factor of 4 . However, if the excitation temperature of the $18 \mathrm{~cm}$ lines is less than the assumed $20 \mathrm{~K}$ (see the discussion in Stacey et al. 1987), the radio column density could be an overestimate. Also, the $18 \mathrm{~cm}$ lines may sample a different component of the envelope as the depth at which the continuum is emitted occurs deeper into the cloud than in the FIR region, and the OVRO synthesised beam $\left(3.25^{\prime}\right)$ is larger than that of ISO. Bieging (1976) estimates that the column density in the negative velocity features is smaller than the positive velocity values by about a factor of four.

The total column density of ${ }^{18} \mathrm{OH}$ over the whole line of sight in our fit was $(1.8 \pm 0.2) \times 10^{14} \mathrm{~cm}^{-2}$ and this compares favourably with previous observations using the Kuiper Airborne Observatory: Lugten et al. (1986) found a total column density of $N\left({ }^{18} \mathrm{OH}\right) \geq 2 \times 10^{14} \mathrm{~cm}^{-2}$.

\section{5. ${ }^{16} \mathrm{OH} /{ }^{18} \mathrm{OH}$ ratio}

Table 1 gives the best fitting ${ }^{16} \mathrm{OH} /{ }^{18} \mathrm{OH}$ ratios. In order to compare with previous measurements of the isotopic ratios, a galactocentric distance must be assigned to each feature. Several of the velocity components have been well established to come from galactic spiral arm and Galactic Centre features such as the Galactic Bar at large negative velocities (Scoville 1972), the $3 \mathrm{kpc}$ expanding arm at $-44 \mathrm{~km} \mathrm{~s}^{-1}$ (e.g. Burke 1965), the $4-5 \mathrm{kpc}$ arm at $-24 \mathrm{~km} \mathrm{~s}^{-1}$ (Menon \& Ciotti 1970) and Sgr B2 (M) itself at $+50-70 \mathrm{~km} \mathrm{~s}^{-1}$ (e.g. Martín-Pintado et al. 1990). Associations for the remaining features have been proposed by Greaves \& Williams (1994). In this case, the 1, 16 and $31 \mathrm{~km} \mathrm{~s}^{-1}$ features would be associated with local spiral arms. However, it has been suggested that there may be a contribution at $0 \mathrm{~km} \mathrm{~s}^{-1}$ by gas associated with Sgr B2 (e.g Gardner et al. 1988).

Figure 5 shows our results plotted against their distances from the Galactic Centre taken from Greaves \& Williams (1994). Compilations of many observations of $\mathrm{H}_{2} \mathrm{CO}$ through the Galaxy indicate that there is a gradient in the ${ }^{16} \mathrm{O} /{ }^{18} \mathrm{O}$ ratio with decreasing values towards the Galactic Centre (Tosi 1982; Wilson \& Rood 1994; Kahane 1994). The best fit gradient found by Wilson \& Rood (1994) is overplotted in Fig. 5 as

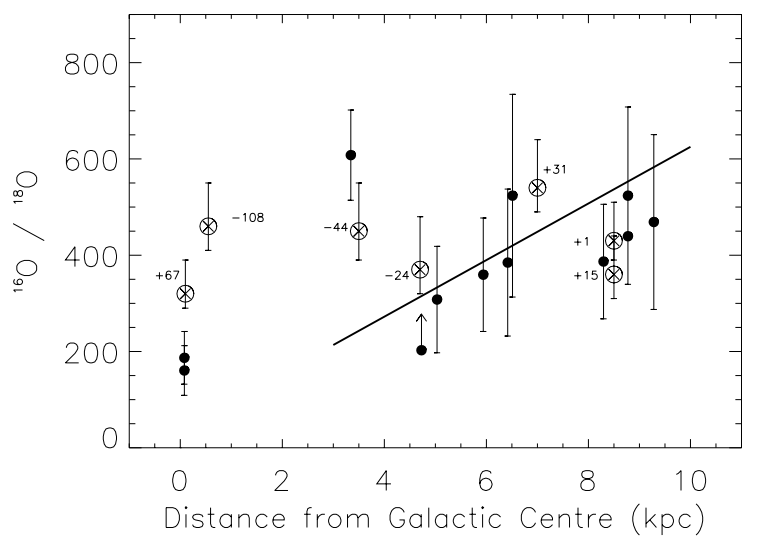

Fig. 5. Values of ${ }^{16} \mathrm{OH} /{ }^{18} \mathrm{OH}$ from Table 1 plotted against distance from Galactic Centre (large circles with cross). The velocity of each component is indicated in $\mathrm{km} \mathrm{s}^{-1}$ next to each point. The compilation of values from Kahane (1994) is plotted for comparison, as well as the best fitting galactic gradient found by Wilson \& Rood (1994).

well as the compilation of $\mathrm{H}_{2} \mathrm{CO}$ and $\mathrm{CO}$ results from Kahane (1994).

The three most uncertain distances are for the local components. At $31 \mathrm{~km} \mathrm{~s}^{-1}$ we find a relatively high value of ${ }^{16} \mathrm{OH} /{ }^{18} \mathrm{OH}$, consistent with its location close to the Sun. At $1 \mathrm{~km} \mathrm{~s}^{-1}$, our value is in good agreement with that found from $\mathrm{OH} \Lambda$-doublet lines of 440 (Bujarrabal et al. 1983). However, both the 1 and $16 \mathrm{~km} \mathrm{~s}^{-1}$ components have rather lower values than might be expected for local gas. The gradient of Wilson $\&$ Rood (1994) predicts a local value of $560 \pm 25$, although it has been measured to be even higher using $\mathrm{HCO}^{+}$absorption by Lucas \& Liszt (1998), giving $672 \pm 110$. This may indicate that the local absorbing gas towards Sgr B2 has atypical isotopic abundances. An anomalously low ${ }^{12} \mathrm{C} /{ }^{13} \mathrm{C}$ ratio has also been found in this gas by Greaves (1995). She rules out an error in the distance estimate and attributes the extra ${ }^{13} \mathrm{C}$ to enrichment by stellar ejecta containing secondary nucleosynthesis products. In this case it would be natural to also find a higher abundance of ${ }^{18} \mathrm{OH}$.

In comparison, the standard Solar System abundance (derived from Standard Mean Ocean Water $)$ is $\left({ }^{16} \mathrm{O} /{ }^{18} \mathrm{O}\right)_{\text {SMOW }}=$ $498.7 \pm 0.1$ (Baertschi 1976). The value in the outer layers of the Sun has been measured to be ${ }^{16} \mathrm{O} /{ }^{18} \mathrm{O}=440 \pm 50$ (Harris et al. 1987) and in the Solar wind, Collier et al. (1998) found ${ }^{16} \mathrm{O} /{ }^{18} \mathrm{O}=450 \pm 130$. This should represent the conditions when the Sun was formed and models predict that it should be higher than the current ratio in the local ISM due to the build up of secondary elements from stellar processing (e.g. Prantzos et al. 1996). In order to explain the low Solar System abundances compared to the ISM, it has been proposed that the Sun was formed closer to the Galactic Centre (Wielen \& Wilson 1997).

In the Galactic Centre, the isotope ratio is generally taken to be 250 (Wilson \& Rood 1994). This low value has been derived from measurements of the $\Lambda$-doublet lines of ${ }^{16} \mathrm{OH}$ and ${ }^{18} \mathrm{OH}$ (e.g. Williams \& Gardner 1981; Whiteoak \& Gardner 1981). However, Bujarrabal et al. (1983) show that the ${ }^{16} \mathrm{OH} /{ }^{18} \mathrm{OH}$ opacity ratio derived from the radio lines is only a good 
measure of the actual abundance ratio in cold clouds with kinetic temperatures $\leq 20 \mathrm{~K}$. This is due to excitation anomalies in the hyperfine levels caused by rotational pumping by the FIR lines. This certainly occurs at the velocity of Sgr B2 as can be seen from the higher energy lines of ${ }^{16} \mathrm{OH}$ which show absorption within the ${ }^{2} \Pi_{3 / 2}$ ladder but emission within the ${ }^{2} \Pi_{1 / 2}$ ladder (Goicoechea \& Cernicharo 2002). This would cause the previously derived isotopic ratios to underestimate the true value. At the velocity of Sgr B2, our fit gives a ratio of $320_{-30}^{+70}$. This appears to confirm the suggestion of Bujarrabal et al. (1983) that the ratio in the Galactic Centre has been underestimated (assuming the same excitation conditions exist for ${ }^{16} \mathrm{OH}$ and ${ }^{18} \mathrm{OH}$ and the ground state ratio accurately reflects the isotopic abundance). We also find a high value for the component attributed to the Galactic Bar $\left(-108 \mathrm{~km} \mathrm{~s}^{-1}\right)$ of $460_{-50}^{+90}$. Bujarrabal et al. (1983) find a ratio of $\simeq 370$ in this component.

In contrast, measurements of other molecules in Sgr B2 seem to agree with the lower radio $\mathrm{OH}$ values. However, none of these methods are easy to directly interpret and so may also underestimate the true value. Both $\mathrm{H}_{2} \mathrm{CO}$ and $\mathrm{CO}$ must be used in a double ratio, relying on an accurate value of ${ }^{12} \mathrm{C} /{ }^{13} \mathrm{C}$. Henkel et al. (1983) calculate ${ }^{16} \mathrm{O} /{ }^{18} \mathrm{O} \sim 200$ from measurements of $\mathrm{H}_{2}^{13} \mathrm{C}^{16} \mathrm{O} / \mathrm{H}_{2}^{12} \mathrm{C}^{18} \mathrm{O}$, although the final value is uncertain due to the correction for radiation trapping in $\mathrm{H}_{2}^{12} \mathrm{C}^{16} \mathrm{O}$ (used to determine the associated ${ }^{12} \mathrm{C} /{ }^{13} \mathrm{C}$ ratio). Observations of $\mathrm{CO}$ indicate a value of $250 \pm 100$ in the Galactic Centre (Penzias 1981) but this may be affected by chemical fractionation of ${ }^{13} \mathrm{C}$ (Penzias 1983). Gardner et al. (1989) have observed $\mathrm{CH}_{3}^{16} \mathrm{OH}$ and $\mathrm{CH}_{3}^{18} \mathrm{OH}$ towards Sgr B2 leading to an isotopic ratio of $210 \pm 40$. This does not include a correction for radiation trapping which would increase the ratio. Nummelin et al. (2000) calculate even lower ratios from $\mathrm{S}^{16} \mathrm{O} / \mathrm{S}^{18} \mathrm{O}\left(120_{-54}^{+58}\right)$ and $\mathrm{S}^{16} \mathrm{O}_{2} / \mathrm{S}^{16} \mathrm{O}^{18} \mathrm{O}\left(112_{-22}^{+26}\right)$. They explain these by assuming that the observed emission originates in the central regions of the Sgr B2 complex where ${ }^{18} \mathrm{O}$ could be enhanced by the ejecta from massive stars. Our FIR absorption measurements trace gas in the outer parts of the Sgr B2 envelope.

Without low values at the Galactic Centre, our results show a much less convincing gradient than that obtained from the $\mathrm{CO}$ and $\mathrm{H}_{2} \mathrm{CO}$ measurements. However, we have low number statistics and the distances of some components from the Galactic Centre are not very well known. There also must be some effect from a real variation in clouds at the same galactocentric distance, as indicated by the anomalous ${ }^{16} \mathrm{OH} /{ }^{18} \mathrm{OH}$ and ${ }^{12} \mathrm{C} /{ }^{13} \mathrm{C}$ at $0 \mathrm{~km} \mathrm{~s}^{-1}$. This means that a larger sample is necessary to accurately confirm the trend through the Galaxy.

\section{Comparison with other species}

Several other related species have been observed in the line of sight clouds towards Sgr B2. Observations of absorption due to the $\mathrm{H}_{2}^{16} \mathrm{O}$ and $\mathrm{H}_{2}^{18} \mathrm{O}$ ground state rotational lines have been made using SWAS (Neufeld et al. 2000). Although $\mathrm{H}_{2}^{16} \mathrm{O}$ completely absorbs the continuum, they calculate column densities in three line of sight velocity ranges from $\mathrm{H}_{2}^{18} \mathrm{O}$. Both $\mathrm{OH}$ and $\mathrm{H}_{2} \mathrm{O}$ are formed by the dissociative recombination of $\mathrm{H}_{3} \mathrm{O}^{+}\left(\mathrm{H}_{3} \mathrm{O}^{+}\right.$is also observed to show absorption in the line of sight clouds; Goicoechea \& Cernicharo 2001).
Table 2. Ratio of our derived ${ }^{16} \mathrm{OH}$ column densities with those of $\mathrm{CH}$ from Polehampton et al. (2005) for each of the velocity components from Table 1.

\begin{tabular}{cc}
\hline \hline $\begin{array}{c}\text { Velocity } \\
\left(\mathrm{km} \mathrm{s}^{-1}\right)\end{array}$ & {$\left[{ }^{16} \mathrm{OH} / \mathrm{CH}\right]$} \\
\hline-108 & $6.2 \pm 6.5$ \\
-82 & $35 \pm 21$ \\
$-52 /-44.0$ & $19 \pm 15$ \\
-24.4 & $28 \pm 11$ \\
+1.1 & $38 \pm 18$ \\
+15.7 & $12 \pm 8$ \\
+31 & $36 \pm 35$ \\
$+52.8 /+66.7$ & $34 \pm 7$ \\
\hline
\end{tabular}

Neufeld et al. (2002) show that if recombination of $\mathrm{H}_{3} \mathrm{O}^{+}$is the only production mechanism, the ratio of $\mathrm{OH}$ to $\mathrm{H}_{2} \mathrm{O}$ gives good constraints on the branching ratios. However, they also show that in warm gas, neutral-neutral reactions can alter the balance. A further effect is the inclusion of grain surface reactions, which can increase the gas-phase abundance of $\mathrm{H}_{2} \mathrm{O}$ whilst $\mathrm{OH}$ is relatively unaffected (Plume et al. 2004).

Using the ortho- $\mathrm{H}_{2}^{18} \mathrm{O}$ column densities from Neufeld et al. (2000) with an ortho-to-para ratio of 3 and our ${ }^{16} \mathrm{O} /{ }^{18} \mathrm{O}$ ratios averaged in their velocity ranges gives $\left[\mathrm{H}_{2} \mathrm{O} / \mathrm{OH}\right]$ ratios of 0.6-1.2 towards Sgr B2. These values are a similar order of magnitude to those previously derived for line of sight absorption features towards W51 ( 0.3; Neufeld et al. 2002) and W49 (0.26, 0.43; Plume et al. 2004), indicating a high branching ratio for $\mathrm{H}_{2} \mathrm{O}$ and/or the necessity of including other processes such as gas-grain interactions. In gas at the velocity of Sgr B2 itself, Goicoechea \& Cernicharo (2002) have derived even higher values of $\left[\mathrm{H}_{2} \mathrm{O} / \mathrm{OH}\right] \sim 1-10$. In this case, there is probably a warm gas component present and neutral-neutral reactions could be important.

In diffuse clouds, $\mathrm{CH}$ has been observed to be tightly correlated with $\mathrm{OH}$, with a column density ratio, $[\mathrm{OH} / \mathrm{CH}] \sim 3$ (e.g. Liszt \& Lucas 2002). Absorption from the ground state rotational transition of $\mathrm{CH}$ was observed as part of the ISO spectral survey towards Sgr B2 and has been fitted in a similar way to the $\mathrm{OH}$ lines (Polehampton et al. 2005). Table 2 shows the ratio of our fitted ${ }^{16} \mathrm{OH}$ column densities those of $\mathrm{CH}$ in each velocity component. These have a large uncertainty but seem to indicate values that could be up to ten times higher than that seen in diffuse clouds. This increased ratio cannot be caused by $\mathrm{CH}$ because the abundances found are very similar to diffuse cloud models (Polehampton et al. 2005). We can estimate the abundance of $\mathrm{OH}$ in the line of sight clouds using molecular hydrogen column densities in the range 4 to $14 \times 10^{21} \mathrm{~cm}^{-2}$ (see Greaves \& Nyman 1996). This leads to $\mathrm{OH}$ abundances $10^{-7}-10^{-6}$ in these features. At the velocity of Sgr B2, Goicoechea \& Cernicharo (2002) find an abundance of $(2-5) \times 10^{-6}$. These abundances are much higher than found in diffuse clouds (models of diffuse clouds predict $X(\mathrm{OH}) \sim 10^{-8}$; van Dishoeck \& Black 1986) and lead to the high $[\mathrm{OH} / \mathrm{CH}] \mathrm{ra}-$ tio. Such high abundances of $\mathrm{OH}$ can be found in PDR regions. 


\section{Summary}

In this paper we have presented an analysis of ${ }^{16} \mathrm{OH}$ and ${ }^{18} \mathrm{OH}$ FIR rotational lines towards Sgr B2. Absorption at different velocities along the line of sight allowed us to calculate the ${ }^{16} \mathrm{OH} /{ }^{18} \mathrm{OH}$ ratio between the Sun and Galactic Centre.

The advantages of this technique for determining the isotopic ratio of oxygen are:

- Chemical fractionation effects are not important for ${ }^{16} \mathrm{OH}$ and ${ }^{18} \mathrm{OH}$. Therefore the molecular column density ratio directly measures the isotope abundance.

- There are optically thin lines present from both species.

- The final ratio does not depend on the value of ${ }^{12} \mathrm{C} /{ }^{13} \mathrm{C}$ as for previous $\mathrm{H}_{2} \mathrm{CO}$ and $\mathrm{CO}$ measurements.

- Uncertainties in the population of hyperfine states do not affect the final ratios (as they do for radio observations of $\Lambda$-doublet transitions).

- Only ground state lines are seen for the line of sight clouds, showing that excitation effects are not important in these clouds.

- All observations come from a single dataset with consistent and stable calibration over lines of both isotopologues.

The main disadvantage is the relatively low spectral resolution compared to the velocity separation of line of sight components. This introduced errors for individual components in the line shape modelling. However, the data used here allowed us to maximise confidence in the observed line shapes by combining several observations and with the good coverage of baselines around the lines.

We find ${ }^{16} \mathrm{OH} /{ }^{18} \mathrm{OH}$ ratios broadly consistent with previous isotopic abundances, although our results do not provide such clear evidence for a gradient of the ratio through the Galaxy (but they do not rule it out). In velocity components associated with the Galactic Centre, we find slightly higher ratios than previous results. This could be due to an underestimate in the previous radio observations of $\mathrm{OH}$ caused by pumping of hyperfine levels by the FIR rotational transitions (see Bujarrabal et al. 1983).

In comparing the total ${ }^{16} \mathrm{OH}$ column densities with those of $\mathrm{H}_{2} \mathrm{O}$ towards $\mathrm{Sgr} \mathrm{B} 2$, we find similar magnitude abundance ratios as have been seen in other lines of sight towards W51 and W49 (Neufeld et al. 2002; Plume et al. 2004). A comparison of ${ }^{16} \mathrm{OH}$ with the column density of $\mathrm{CH}$ shows a wide scatter and large error, but appears to indicate higher ratios that previously observed in diffuse clouds.

This study shows that FIR rotational lines are an extremely useful tool for examining the variation of isotopic ratios in the ISM. The column densities of $\mathrm{OH}$ can be accurately derived as the method avoids the anomalous excitation that may be present in radio $\Lambda$-doublet lines. Ideally, future observations at higher spectral resolution are required to improve on the results. This may be possible using future telescopes such as SOFIA.

Acknowledgements. We would like to thank R. Laing, J. Hatchell, T. Wilson and F. van der Tak for helpful discussions, and the referee, C. Kahane, for several useful suggestions. The ISO Spectral Analysis Package (ISAP) is a joint development by the LWS and SWS Instrument Teams and Data Centres. Contributing institutes are CESR, IAS, IPAC, MPE, RAL and SRON. LIA is a joint development of the ISO-LWS Instrument Team at the Rutherford Appleton Laboratory (RAL, UK - the PI Institute) and the Infrared Processing and Analysis Center (IPAC/Caltech, USA).

\section{Appendix A: Observations and wavelengths}

Table A.1. Log of the observations used.

\begin{tabular}{lccccc}
\hline \hline Transition & $\begin{array}{c}\text { Observing } \\
\text { Mode }\end{array}$ & $\begin{array}{c}\text { ISO } \\
\text { TDT } \\
\text { Number }\end{array}$ & $\begin{array}{c}\text { LWS } \\
\text { detector }\end{array}$ & $\begin{array}{c}\text { Spectral } \\
\text { resolution } \\
\left(\mathrm{km} \mathrm{s}^{-1}\right)\end{array}$ & $\begin{array}{c}\text { Repeated } \\
\text { scans }\end{array}$ \\
\hline${ }^{16} \mathrm{OH}$ & & & & & \\
${ }^{2} \Pi_{3 / 2} J=5 / 2-3 / 2(119 \mu \mathrm{m})$ & L03 & 50601013 & LW2 & 33 & 3 \\
\hline${ }^{2} \Pi_{1 / 2}{ }^{2} \Pi_{3 / 2}$ & L03 & 50400823 & SW2 & 61 & 3 \\
$J=3 / 2-3 / 2(53 \mu \mathrm{m})$ & L03 & 50601013 & SW2 & 61 & 3 \\
& L03 & 50700707 & SW2 & 61 & 3 \\
& L03 & 50800317 & SW2 & 61 & 3 \\
\hline${ }^{2} \Pi_{1 / 2}{ }^{2} \Pi_{3 / 2}(79 \mu \mathrm{m})$ & L03 & 50900521 & SW5 & 40 & 3 \\
$J=1 / 2-3 / 2(703$ & 50600814 & SW5 & 40 & 3 \\
& L03 & 50600603 & SW4 & 40 & 3 \\
& L03 & 50700208 & SW4 & 40 & 3 \\
& L03 & 83800606 & SW4 & 40 & 4 \\
& L03 & 50600506 & SW4 & 40 & 3 \\
\hline${ }^{18} \mathrm{OH}$ & & & & & \\
${ }^{2} \Pi_{3 / 2} J=5 / 2-3 / 2(120 \mu \mathrm{m})$ & L03 & 50601013 & LW2 & 33 & 3 \\
& L03 & 50700610 & LW2 & 33 & 3 \\
& L04 & 49401705 & LW2 & 33 & 15 \\
& L04 & 46201118 & LW2 & 33 & 11 \\
& L04 & 46900332 & LW2 & 33 & 14 \\
\hline
\end{tabular}


Table A.2. Wavelengths and Einstein coefficients $\left(A_{i j}\right)$ for ${ }^{16} \mathrm{OH}$ and ${ }^{18} \mathrm{OH}$ averaged over hyperfine structure. The ${ }^{16} \mathrm{OH}$ wavelengths are from Brown et al. (1982) and Varberg \& Evenson (1993) and the Einstein coefficients were calculated from the line strengths of Brown et al. (1982). The ${ }^{18} \mathrm{OH}$ wavelengths are from Morino et al. (1995) with Einstein coefficients calculated from the line strengths of Comben et al. (1986).

\begin{tabular}{lcc}
\hline \hline Transition & $\begin{array}{c}\text { Wavelength } \\
(\mu \mathrm{m})\end{array}$ & $\begin{array}{c}A_{i j} \\
\left(\mathrm{~s}^{-1}\right)\end{array}$ \\
\hline${ }^{16} \mathrm{OH}$ & & \\
$J=3 / 2-3 / 2^{a}$ & 53.2615 & 0.04559 \\
& 53.3512 & 0.04481 \\
$J=1 / 2-3 / 2^{a}$ & 79.1176 & 0.03575 \\
& 79.1812 & 0.03545 \\
\hline${ }^{18} \mathrm{OH}$ & & \\
$J=5 / 2-3 / 2$ & 119.9651 & 0.1364 \\
& 120.1718 & 0.1357 \\
\hline${ }^{a} \mathrm{Cross}$ ladder transition. ${ }^{2} \Pi_{1 / 2}{ }^{2} \Pi_{3 / 2}$
\end{tabular}

\section{References}

Baertschi, P. 1976, Earth and Planetary Science Letters, 31, 341

Bieging, J. H. 1976, A\&A, 51, 289

Brown, J. M., Schubert, J. E., Evenson, K. M., \& Radford, H. E. 1982, ApJ, 258, 899

Bujarrabal, V., Cernicharo, J., \& Guélin, M. 1983, A\&A, 128, 355

Burke, B. F. 1965, ARA\&A, 3, 275

Ceccarelli, C., Baluteau, J.-P., Walmsley, M., et al. 2002, A\&A, 383, 603

Clegg, P. E., Ade, P. A. R., Armand, C., et al. 1996, A\&A, 315, L38

Collier, M. R., Hamilton, D. C., Gloeckler, G., et al. 1998, J. Geophys. Res., 103, 7

Comben, E. R., Brown, J. M., Steimle, T. C., Leopold, K. R., \& Evenson, K. M. 1986, ApJ, 305, 513

Gardner, F. F., Boes, F., \& Winnewisser, G. 1988, A\&A, 196, 207

Gardner, F. F., Whiteoak, J. B., Reynolds, J., Peters, W. L., \& Kuiper, T. B. H. 1989 , MNRAS, $240,35 \mathrm{P}$

Garwood, R. W., \& Dickey, J. M. 1989, ApJ, 338, 841

Goicoechea, J. R., \& Cernicharo, J. 2001, ApJ, 554, L213

Goicoechea, J. R., \& Cernicharo, J. 2002, ApJ, 576, L77

Goicoechea, J. R., Roderíguez-Fernández, N. J., \& Cernicharo, J. 2004, ApJ, 600, 214

Goicoechea, J. R., Martín-Pintado, J., \& Cernicharo, J. 2005, ApJ, 619,291

Greaves, J. S. 1995, MNRAS, 273, 918

Greaves, J. S., \& Nyman, L. 1996, A\&A, 305, 950

Greaves, J. S., \& Williams, P. G. 1994, A\&A, 290, 259

Gry, C., Swinyard, B., Harwood, A., et al. 2003, ISO Handbook Volume III (LWS), Version 2.1, ESA SAI-99-077/Dc

Harris, M. J., Lambert, D. L., \& Goldman, A. 1987, MNRAS, 224, 237

Henkel, C., Wilson, T. L., Walmsley, C. M., \& Pauls, T. 1983, A\&A, 127,388

Kahane, C. 1994, in Nuclei in the Cosmos III: 3rd International Symposium on Nuclear Astrophysics, AIP Conf. Proc., 327, 19
Langer, W. D., Graedel, T. E., Frerking, M. A., \& Armentrout, P. B. 1984, ApJ, 277, 581

Lim, T. L., Hutchinson, G., Sidher, S. D., et al. 2002, SPIE, 4847, 435

Liszt, H., \& Lucas, R. 2002, A\&A, 391, 693

Lloyd, C. 2002, in The Calibration Legacy of the ISO Mission, ESA SP-481, 399

Lucas, R., \& Liszt, H. 1998, A\&A, 337, 246

Lugten, J. B., Stacey, G. J., \& Genzel, R. 1986, BAAS, 18, 1007

Martín-Pintado, J., de Vicente, P., Wilson, T. L., \& Johnston, K. L. 1990, A\&A, 236, 193

McGee, R. X., Gardner, F. F., \& Sinclair, M. W. 1970, PASA, 1, 334

Mehringer, D. M., Palmer, P., \& Goss, W. M. 1995, ApJS, 97, 497

Melnick, G. J., Stacey, G. J., Genzel, R., Lugten, J. B., \& Poglitsch, A. 1990, ApJ, 348, 161

Menon, T. K., \& Ciotti, J. E. 1970, Nature, 227, 579

Morino, I., Odashima, H., Matsushima, F., Tsunekawa, S., \& Takagi, K. 1995, ApJ, 442, 907

Neufeld, D. A., Ashby, M. L. N., Bergin, E. A., et al. 2000, ApJ, 539, L111

Neufeld, D. A., Kaufman, M. J., Goldsmith, P. F., Hollenbach, D. J., \& Plume, R. 2002, ApJ, 580, 278

Nummelin, A., Bergman, P., Hjalmarson, A., et al. 2000, ApJS, 128, 213

Penzias, A. A. 1981, ApJ, 249, 518

Penzias, A. A. 1983, ApJ, 273, 195

Plume, R., Kaufman, M. J., Neufeld, D. A., et al. 2004, ApJ, 605, 247

Polehampton, E. T. 2002, Ph.D. Thesis, Oxford University

Polehampton, E. T., Baluteau, J.-P., Ceccarelli, C., Swinyard, B. M., \& Caux, E. 2002a, A\&A, 388, L44

Polehampton, E. T., Swinyard, B. M., \& Baluteau, J.-P. 2002b, in Exploiting the ISO Data Archive, ESA SP-511, 205

Polehampton, E. T., Brown, J. M., Swinyard, B. M., \& Baluteau, J.-P. 2003, A\&A, 406, L47

Polehampton, E. T., Menten, K. M., Brünken, S., Winnewisser, G., \& Baluteau, J.-P. 2005, A\&A, 431, 203

Prantzos, N., Aubert, O., \& Adouze, J. 1996, A\&A, 309, 760

Press, W. H., Teukolsky, S. A., Vetterling, W. T., \& Flannery, B. P. 1992, Numerical Recipes in Fortran 77, 2nd ed. (Cambridge: Cambridge University Press)

Scoville, N. Z. 1972, ApJ, 175, 127

Spitzer, L. 1978, Physics of the Interstellar Medium (New York: John Wiley and Sons)

Stacey, G. J., Lugten, J. B., \& Genzel, R. 1987, ApJ, 313, 859

Sturm, E., Bauer, O. H., Lutz, D., et al. 1998, in Astronomical Data Analysis Software and Systems VII, ASP Conf. Ser., 145, 161

Swinyard, B. M., Burgdorf, M. J., Clegg, P. E., et al. 1998, SPIE, 3354, 888

Tosi, M. 1982, ApJ, 254, 699

van Dishoeck, E. F., \& Black, J. H. 1986, ApJS, 62, 109

Varberg, T. D., \& Evenson, K. M. 1993, J. Molec. Spectrosc., 157, 55

Vastel, C., Polehampton, E. T., Baluteau, J.-P., et al. 2002, ApJ, 581, 315

Whiteoak, J. B., \& Gardner, F., F. 1981, MNRAS, 197, P39

Whiteoak, J. B., \& Gardner, F. F. 1975, PASA, 2, 360

Wielen, R., \& Wilson, T. L. 1997, A\&A, 326, 139

Williams, D. R., \& Gardner, F. F. 1981, PASP, 93, 82

Wilson, T. L., \& Matteucci, F. 1992, A\&ARv, 4, 1

Wilson, T. L., \& Rood, R. T. 1994, ARA\&A, 32, 191 